\title{
A cross-sectional study of the reporting quality of pilot or feasibility trials in high-impact anesthesia journals
} Étude transversale sur les rapports d'études pilotes et d'études de
faisabilité dans les journaux d'anesthésie à facteur d'impact élevé

\author{
Harsha Shanthanna, MD, MSc (1) • Alka Kaushal, MBBS • Lawrence Mbuagbaw, PhD • \\ Rachel Couban, MISt $\cdot$ Jason Busse, PhD $\cdot$ Lehana Thabane, PhD
}

Received: 13 November 2017/Revised: 24 April 2018/Accepted: 14 May 2018/Published online: 9 August 2018

(C) Canadian Anesthesiologists' Society 2018

\begin{abstract}
Purpose Pilot trials inform the design and conduct of larger scale trials. Using the Consolidated Standards of Reporting Trials (CONSORT) pilot extension guidelines, we assessed reporting quality in five high-impact anesthesia journals and explored factors associated with reporting quality.

Methods The five highest-impact anesthesia journals were screened for randomized-controlled trials published as pilot or feasibility trials between 2006 and 2016. A pair of reviewers independently screened citations, extracted data, and assessed reporting quality using the CONSORT pilot trial extension checklists for abstracts and full texts. We reported the percentage adherence for each item, along with the median [interquartile range (IQR)] or mean (standard deviation [SD]) for all items. The factors considered to influence reporting were: 1) trial registration, 2) industry funding, 3) trial identification as
\end{abstract}

H. Shanthanna, MD, MSc ( $\square) \cdot$ L. Thabane, PhD

Department of Anesthesia, St. Joseph's Healthcare Hamilton,

McMaster University, 50 Charlton Avenue East, Hamilton, ON

L8N 4A6, Canada

e-mail: shanthh@mcmaster.ca

H. Shanthanna, MD, MSc - A. Kaushal, MBBS - L. Mbuagbaw, $\mathrm{PhD}$. J. Busse, $\mathrm{PhD} \cdot \mathrm{L}$. Thabane, $\mathrm{PhD}$

Department of Health Research Methods, Evidence, and Impact, McMaster University, Hamilton, ON, Canada

A. Kaushal, MBBS · R. Couban, MISt · J. Busse, PhD

The Michael G. DeGroote Institute for Pain Research and Care, McMaster University, Hamilton, ON, Canada

L. Mbuagbaw, PhD $\cdot$ L. Thabane, $\mathrm{PhD}$

Biostatistics Unit, St Joseph's Healthcare, Hamilton, ON,

Canada a pilot or feasibility in the title or abstract, 4) primary objective as "feasibility", and 5) the specific journal. The association was estimated using generalized estimating equations and reported as incidence rate ratios with $99 \%$ confidence intervals.

Results Of 364 citations, 58 articles were eligible. The median [IQR] number of CONSORT abstract items reported was 5 [4-7], and the mean (SD) number of full text items reported was 13 (5). Significantly poor reporting was associated with "not registering the trial" (both abstracts and full texts), "trial not identified as a pilot" (abstracts), and "using clinical hypothesis as the primary objective" (full texts).

Conclusion The reporting quality of pilot trials published in leading anesthesia journals is poor. Journal editorial boards can encourage improved reporting by supporting adherence to the CONSORT extension for pilot trials.

\section{Résumé}

Objectif Les études pilotes renseignent sur la conception et la réalisation d'essais à plus grande échelle. Nous avons utilisé les lignes directrices sur l'extension aux études pilotes $d u$ regroupement des normes relatives aux rapports d'étude (CONSORT) pour évaluer la qualité des rapports dans cinq journaux d'anesthésie à fort impact et explorer les facteurs associés à la qualité des rapports.

Méthodes Nous avons recherché les publications d'essais contrôlés randomisés d'études pilotes ou de faisabilité dans les cinq journaux d'anesthésie ayant les facteurs d'impact les plus élevés entre 2006 et 2016. Deux réviseurs indépendants ont examiné les citations et les données extraites; ils ont évalué la qualité des rapports (résumés et textes complets) en utilisant les listes de vérification CONSORT pour l'extension aux études pilotes. Nous 
avons fourni le pourcentage d'adhésion à chaque critère avec les valeurs médianes (écart interquartile [EIQ]) ou moyennes (écart-type [ET]) pour tous les critères. Les facteurs qui ont été considérés comme influençant les rapports étaient les suivants: 1) l'enregistrement de l'étude, 2) le financement par l'industrie, 3) l'identification de l'essai en tant qu'étude pilote ou de faisabilité dans son titre, 4) l'objectif principal indiquant "faisabilité », et 5) le journal dans lequel paraît la publication. L'association a été estimée au moyen d'équations d'estimation généralisée et décrite sous forme d'incidences avec des intervalles de confiance à $99 \%$.

Résultats Sur 364 références, 58 articles ont été retenus. Le nombre médian (EIQ) de critères dans les résumés CONSORT rapportés était de 5 (4 à 7) et le nombre moyen (ET) de critères rapportés dans les textes complets était de 13 (5). Un rapport de qualité significativement médiocre était associé au "non-enregistrement de l'essai » (pour les résumés comme pour les textes complets), à la "non-identification de l'essai en tant qu'étude pilote" (résumés) et à "l'utilisation d'une hypothèse clinique comme objectif principal »(textes complets).

Conclusion La qualité des rapports des études pilotes publiés dans les principaux journaux d'anesthésie est médiocre. Les Comités éditoriaux des journaux peuvent encourager l'amélioration des rapports en soutenant l'adhésion aux critères CONSORT d'extension aux études pilotes.

Pilot trials are considered preliminary studies performed to inform methodologic considerations to design a subsequent study that is larger and confirmatory. ${ }^{1,2}$ Most consider a pilot study to be a feasibility test of the methods and procedures $^{1-3}$ and as such do not address clinical efficacy. ${ }^{4}$ Accurate, transparent, and consistent reporting indicates scientific integrity and minimizes the potential for bias and distortion of effect estimates associated with incomplete reporting. The Consolidated Standards of Reporting Trials (CONSORT) framework was introduced to improve reporting of randomized-control trials (RCTs). ${ }^{5}$ Nevertheless, an assessment of reporting in anesthesia literature, comparing before and after CONSORT implementation, did not show much improvement. ${ }^{6}$ It is essential that pilot studies are reported to avoid redundancy to make appropriate use of resources and funds and to allow the possibility of including the results in an appropriate meta-analysis. ${ }^{7,8}$ Despite the relative increase in their publication, ${ }^{9}$ their reporting quality is often poor. ${ }^{7}$ In response, a framework for reporting pilot studies based on the CONSORT statement was developed and published in 2016. ${ }^{10}$ This includes separate guidance statements and checklists for the abstract (16 items) and full text of the manuscript (26 items). As suggested, for the purposes of this study, "pilot" and "feasibility" will be used interchangeably and the included studies will be referred to as pilot trials. To assess standards of reporting, previous studies have looked at adherence to CONSORT RCT guidelines, ${ }^{11}$ and some have explored predictive factors. ${ }^{12-14}$ Since no studies have evaluated the reporting standards of pilot trials in the anesthesia literature, we decided to look at studies published between 2006 and 2016 (prior to the CONSORT pilot trial extension) and evaluate their reporting in reference to the CONSORT pilot trial extension checklists for abstracts and full texts. Our objectives were to assess the reporting quality of 1) abstracts and 2) full texts in five leading anesthesia journals and explore potential factors associated with reporting quality.

\section{Methods}

This is a cross-sectional study of pilot trials published in leading anesthesia journals. This manuscript adheres to the STROBE statement and checklist for cross-sectional studies.

Study screening and selection

We identified the top five highest impact journals in the field of anesthesiology and pain according to the 2015 journal citation reports. ${ }^{15}$ The impact factor (IF) refers to an index number assigned and calculated by Thomson Reuters as the number of citations received in a specific year for articles published in that journal during the two preceding years divided by the total number of articles published in that journal during the two preceding years. ${ }^{16}$ Our search strategy was designed by an experienced librarian (R.C.) using the OVID platform and was guided by the terms "pilot", "feasibility", or "preliminary" studies (Appendix 1). The initial search was performed on 6 October 2016 to look for studies published from 2012 to 2016 (last five years). Since our first search potentially led to only 38 full-text articles, we expanded our search on 26 October 2016 to expand the search to include articles from the last ten years. We included all pilot trials that were designed to assess the feasibility, safety, or acceptability of an intervention and that included a randomization procedure. We excluded trials on animals and pharmacokinetic studies. No language exclusion criterion was applied. For our review, study selection based on the term "feasibility" was challenging. In 
general, the term "feasibility" reflects the "ease" or "possibility" of either an intervention or method. For example, we had to separate studies that were aimed at looking at the ease of ultrasound use for injection or visualization or time taken with a fibreoptic bronchoscope compared with other methods of intubation. Such studies were excluded as they were not expected to lead to a subsequent larger trial. The study selection process was carried out in duplicate by two independent reviewers (A.K. and H.S.), both trained in health research methods. The title and abstract screening was conducted using a free online software, "rayyan" (https://rayyan.qcri.org/). The full-text screening was done independently and in duplicate. The agreement on full-text screening was reported using a kappa $(\mathrm{k})$ statistic and interpreted as poor if $\mathrm{k} \leq$ 0.2 , fair if $0.21 \leq \mathrm{k} \leq 0.4$, moderate if $0.41 \leq \mathrm{k} \leq 0.6$, substantial if $0.61 \leq \mathrm{k} \leq 0.8$, and good if $\mathrm{k}>0.8 .{ }^{17}$ Disagreement was resolved by consensus or arbitration by a third reviewer (L.M.).

\section{Data abstraction}

Data abstraction was carried out by two independent reviewers (A.K. and H.S.) using extraction forms created in Microsoft Excel, 2016 (Microsoft, Redmond, WA, USA). Extracted items included the study characteristics; reason for identifying a study as a pilot trial; study conclusion as feasible, not feasible, or unclear; and CONSORT reporting items separately for the abstract and full text, as guided by the recently published CONSORT pilot trial extension statement. ${ }^{10}$ Reviewers were provided with clear and explicit rules to judge each CONSORT item as either "reported" or "not reported" (Appendix 3). All items with sub-items were collapsed into a single item allowing for a single decision to be made as "reported" or "not reported" for each item. The extraction forms were piloted to ensure consistency until an agreement score of $\mathrm{k}>0.8$ was achieved for at least three consecutive studies. The total number of items assessed for abstracts was 15; the item "recruitment" was not considered as it was relevant only to conference abstracts. ${ }^{10}$ The total number of items assessed for full texts was 24; the items "ancillary analyses (results of any other analyses performed that could be used to inform the future definitive trial)" and "trial protocol (where the pilot trial protocol can be accessed, if available)" were omitted from the total items as they were optional and difficult to judge.

Study outcomes

Our primary outcomes were to assess the reporting quality of 1) abstracts and 2) full texts using the CONSORT extension for pilot trials and secondarily as explored factors associated with reporting quality.

\section{Predictor variables}

Apart from including the following four variables as either present or absent in all studies-1) industry funding; 2) clinical trial registration as a pilot trial; 3) primary outcome or objective as a feasibility outcome or objective; 4) trial identified as either "pilot" or "feasibility" in either its title and/or abstract-we considered the journal name as a grouping factor. We hypothesized that not registering the trial as a pilot trial, not reporting the feasibility target as a primary objective or outcome, and not identifying the trial as a pilot trial in its title and/or abstract would be associated with poor reporting quality. Information on industry funding was considered when it was identified with any part of study reporting. For registration, we checked whether each trial was registered within ClinicalTrials.gov or the International Clinical Trials Registry Platform (ICTRP)-World Health Organization registry. Further, whenever registered we also checked whether they were in fact registered as a pilot or feasibility trial and if there were any amendments to that effect. We hypothesized that a higher journal impact factor and industry funding would be associated with better reporting quality.

\section{Statistical analysis}

Important study characteristics were tabulated along with their reported frequencies and percentages. For continuous outcomes, mean (SD) or median [interquartile range (IQR)], was reported. Categorical data, such as the number of studies reporting a particular item, were reported as counts and percentages. All included trials were assessed for completeness of CONSORT items reporting (abstract $=15$ and full text $=24)$. Normality of the distribution was tested using the Kolmogorov-Smirnov (KS) test (at a significance of $P<0.05$ ) and visual inspection of Q-Q plots.

Individual abstract and full-text items, and their percentage reporting across studies, were represented with bar diagrams. We explored the association of predictive factors with the total number of CONSORTrecommended abstract and full-text items reported using a generalized estimating equations model. Our exploratory variables included four categorical variables and the specific journal (grouping factor). The articles from each journal can be clustered and correlated because of the journal policy, word limitations, and other publication considerations. Nevertheless, since we do not know the structure of this correlation, we assumed the working 
correlation matrix as unstructured, with the covariance matrix for the robust estimator and not the model-based estimator. With a generalized linear modelling (genlin), we used the Poisson log-linear function after checking for Poisson distribution, by KS test (at a significance of $P \leq$ 0.05 ) for Poisson distribution, and also comparing the mean and variance of the dependent variables. Under the model, we considered the above-specified covariates and looked for main effects. Under parameter estimation, we used a hybrid method with Fisher (1): scale $=1$, maximum iterations $=100$ and maximum step-halving $=5$, and parameter convergence $=1 \mathrm{E}-006$ (absolute). Under statistics, we looked for analysis type III for Wald Chisquare statistics with a confidence interval (CI) specified at 99\% and full log quasi-likelihood function. Although we had more than five counts (dependent variable) per covariate, there is potential for a false discovery rate, so we considered significance at alpha $(\alpha) \leq 0.01$ and estimated the incidence rate ratios (IRR), along with their 99\% CI. We explored variance inflation factors (VIFs), with a threshold of 10 , to assess collinearity between predictors. ${ }^{18}$ Microsoft Excel 2016 and IBM SPSS 24.0.0.0 (2016; IBM Corporation, Armonk, NY, USA) were used to perform the statistical analysis.

\section{Sample size}

We did not estimate the actual sample size required. To get a fairly unbiased estimate we decided to obtain at least 50 studies as a convenience sample.

\section{Results}

The five highest-impact anesthesia journals identified as per the 2015 journal citation reports (ranked from higher to lower) were British Journal of Anaesthesia (BJA), Pain, Anesthesiology, Anesthesia \& Analgesia (AA), and Anaesthesia; IFs ranged from 5.61 to 3.79. Our search identified a total of 364 potential citations, of which 83 were selected for full-text review and 58 were eligible for data extraction (Fig. 1). The reviewers agreed on 79 of the 83 articles leading to a kappa of 0.91 (95\% CI, $0.81 \%$ to 1.00). Of 58 articles, three were published as letters to editors $^{19-21}$ and the rest as original articles. Important study characteristics are highlighted in Table 1. Two of the five journals (BJA and AA) contributed $62 \%$ of the included articles. The median [IQR] sample size was 43 (30-76). In 42 studies $(72 \%)$ the rationale for reporting the study as pilot or feasibility was unclear or not provided (Table 1).
Primary outcomes

\section{CONSORT ABSTRACT REPORTING}

We considered a total of 55 articles for the reporting quality of abstracts (by excluding the three trials reported as letters). Tests for normality indicated that they were not normally distributed. The median [IQR] number of items reported was 5 [4-7]. All CONSORT recommended items except four (trial design, participants, intervention, and randomization) were reported in less than $40 \%$ of studies (Fig. 2). "Trial funding" was observed to be the least commonly reported item. For CONSORT full-text reporting, we included all 58 articles for assessment. The reporting of CONSORT items was normally distributed (KS test significance 0.200 ) with a mean (SD) of 13 (5). Among CONSORT items, we observed that "ethical approval" was the most commonly reported item, while "title and abstract" was the least commonly reported (Fig. 3). Reporting of individual studies is provided in Appendix 2.

\section{Secondary outcomes (Table 2)}

None of the VIFs were $>10$ in our regression models, suggesting no major collinearity among independent factors. The IRR is interpreted similarly to the odds ratio. ${ }^{22}$ For example, an IRR of 1.0 for "trial registration" would mean there is no difference in reporting, whereas $>1$ would mean there is increased odds of better reporting with trial registration. For CONSORT abstract reporting, our regression analysis showed that "not registering the trial as a pilot trial" (IRR, $0.68 ; 99 \% \mathrm{CI}, 0.54$ to $0.87 ; P<0.001$ ) and "not identifying it as a pilot trial in the title or abstract" (IRR, $0.69 ; 99 \% \mathrm{CI}, 0.54$ to $0.89 ; P<0.001)$ were significantly associated with poor reporting quality. For CONSORT fulltext reporting, "not registering the trial as a pilot trial" (IRR, $0.67 ; 99 \%$ CI, 0.54 to $0.82 ; P<0.001)$ and "using clinical hypothesis testing as the primary objective and/or outcome" (IRR, 0.77; 99\% CI, 0.63 to $0.93 ; P=0.001$ ) were significantly associated with poor reporting quality (Table 2).

\section{Discussion}

Summary of findings

We found that that the reporting quality of pilot trials is poor for both the abstract and full text or reports published in leading anesthesia journals. For abstracts, the median [IQR] items reported was 5 [4-7], and for full texts the mean (SD) items reported was 13 (5). A significant association of poor reporting was observed with "not 
Fig. 1 Study flow chart
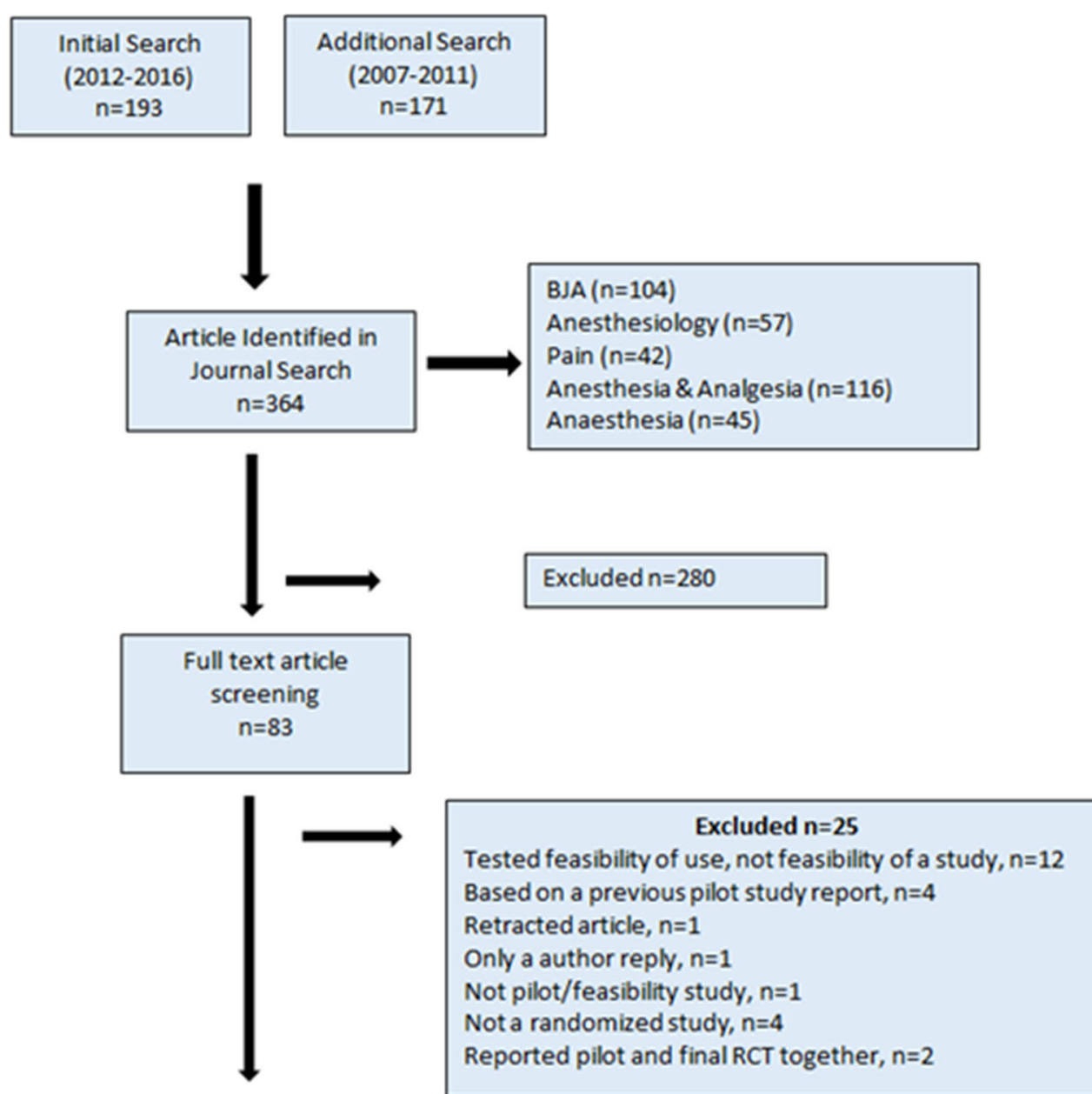

Final selection for

extraction and analysis

$\mathrm{n}=58$

registering as a pilot trial" and "not identifying as a pilot trial in their title or abstract" and with "not registering as a pilot trial" and "reporting clinical hypothesis testing as the primary outcome/objective" for abstracts and full texts, respectively.

To measure the quality of reporting of published RCTs, the CONSORT RCT statement and its checklist have been

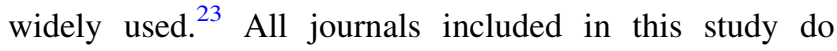
endorse CONSORT guidelines as per the CONSORT website. ${ }^{24} \mathrm{~A}$ recent study looking at the top 11 anesthesia journals and their CONSORT adherence of all items among 319 RCTs reported a median [IQR] compliance rate of $60 \%$ [22.9-88.9\%]. They also observed a weak correlation between adherence to CONSORT items and the number of citations. ${ }^{25}$ Similar observations have been made in other studies in general medical journals ${ }^{26}$ and specialty journals. ${ }^{27}$ While previous studies have evaluated specific elements of reporting, the present study uses the entire CONSORT pilot extension statement and checklists to assess the reporting quality of pilot trials. Outside of anesthesia, other published studies on pilot trials have only looked at specific items of pilot study reporting. Arian et al. studied pilot trial reporting in seven major medical journals published between 2007-2008. ${ }^{4}$ Out of 54 studies, 26 were studies of interventions, of which $86 \%$ incorporated hypothesis testing and $62 \%$ included randomization. Shanyinde et al. looked at a sample of 50 pilot trials among 3,652 publications of pilot RCTs identified within the MEDLINE and EMBASE databases between 20002009. Only 56\% articles discussed adequate methodologic issues and only $18 \%$ discussed future trials. ${ }^{9}$

In our study, items such as "title and abstract", "background and objectives", "outcomes", and "statistical methods" were observed to be appropriately reported in $<20 \%$ of studies, as the majority focused on hypothesis testing as their primary outcome (Fig. 3). Duffet et al. studied pilot RCTs published in pediatric critical care literature and found only 32 trials up to July 2014. They 
Table 1 Characteristics of included studies $(n=58)$

\begin{tabular}{lr}
\hline Years published & 2007-2016
\end{tabular}

Number of articles in each journal [IF] $(n=58)$

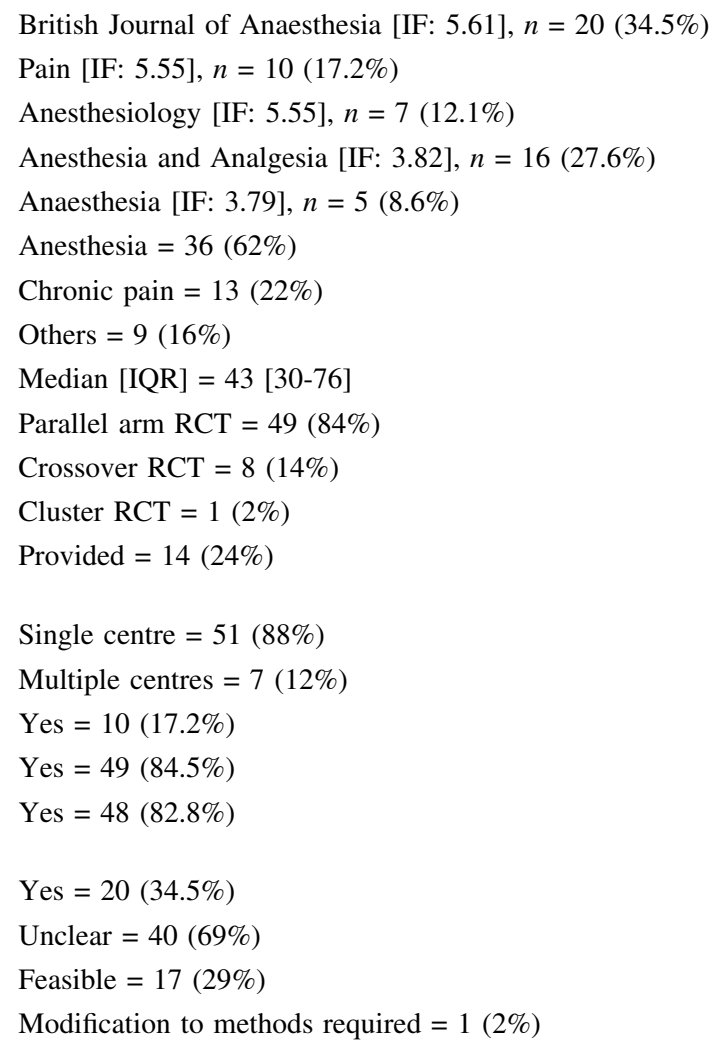

Studies providing appropriate rationale for pilot study (as feasibility, proof concept, or phase 2) $(n=58)$

Studies categorized based on number of centres $(n=58)$

Industry funding among studies reporting funding $(n=49)$

Studies with pilot or feasibility indicated in title or abstract $(n=58)$

Studies with primary objective or primary outcome

as clinical hypothesis testing $(n=58)$

Studies with trial registration as pilot or feasibility study $(n=58)$

Did the results suggest feasibility for a full trial? $(n=58)$

$\mathrm{IF}$ = impact factor; IQR = interquartile range; $\mathrm{RCT}$ = randomized-controlled trial

also observed that the majority of those trials focused on clinical outcomes and uncommonly reported explicit feasibility outcomes. ${ }^{28}$ This finding is similar to our study, wherein we also observed a significant association of poor reporting when a clinical hypothesis was tested as their primary outcome. Possibly as a consequence of misdirected objectives, we also observed that 40/58 trials were rated as "unclear" for their conclusions on the feasibility of a larger study (Table 1).

In our study, poor reporting was associated with "not registering the trial as a pilot". Registration improves the quality of reporting and makes the trial information publicly available, thereby making the authors accountable, and helps to reduce publication bias and selective reporting. ${ }^{29,30}$ It has been observed that registered trials tend to have a low risk of bias for random sequence generation, allocation concealment, and selective reporting, but not blinding or incomplete outcome data. ${ }^{31}$ Nine trials did not identify their study as a pilot in either their title or abstract. As the title and abstract are the items freely accessible to readers, it is important to be as explicit as possible. For example, Bakri et al. noted their trial to be pilot only in their discussion section. ${ }^{32}$ A clear rationale to identify as a pilot trial was unavailable in $76 \%$ of the trials
(Table 2). We also think it is possible that many were identified so because of the small sample size. It is important that one distinguishes between a small-sized trial, whose estimate of effect can have a large boundary of $\mathrm{CI}$ and hence uncertainty, from a pilot study (large or small is relative) that should be powered or conducted to test the study methods and not the clinical hypothesis. Our study is not without limitations. Selection of studies only from the five journals with the highest IF may not reflect the true picture in anesthesia. Moreover, there are inherent limitations in considering IF as a metric of a journal's quality. It has been observed that citations can be increased by promoting more editorial letters, proportionately more review articles compared original research articles, and by self citations. ${ }^{16}$ Nevertheless, our pre-study preparatory search revealed relatively fewer pilot trials in low-impact anesthesia journals. Although we did not approach the individual journal editors to enquire about their policy on publication of pilot trials, it could be important as observed by Arain $e t a l .{ }^{4}$ They note that, while some journal editors did not encourage them, others considered them less rigorous, and some considered them when trials did not include a sample size calculation. ${ }^{4}$ The item of "sample size" was difficult to judge. Although sample size 


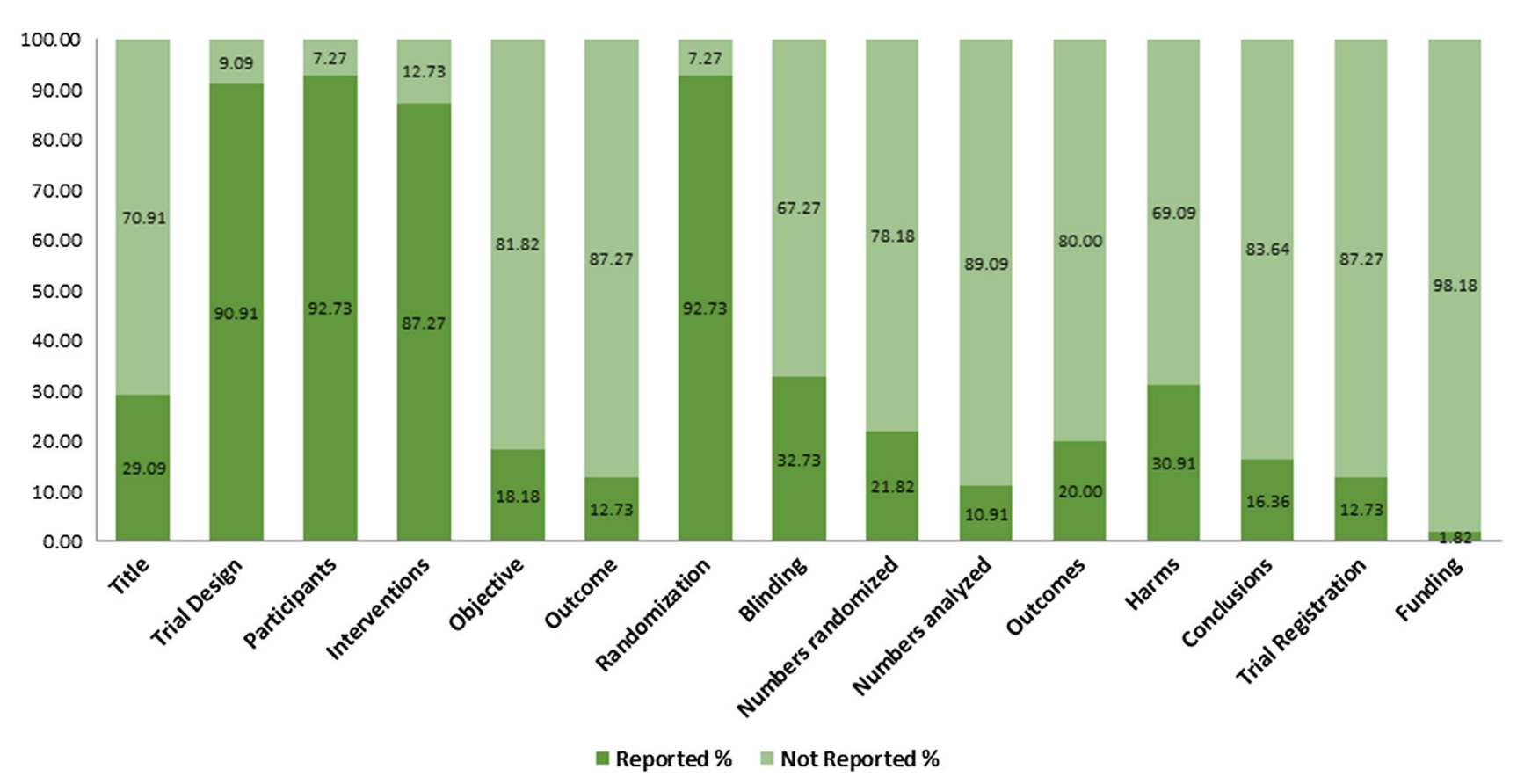

Fig. 2 Consolidated Standards of Reporting Trials pilot extension abstract items reported in percentages

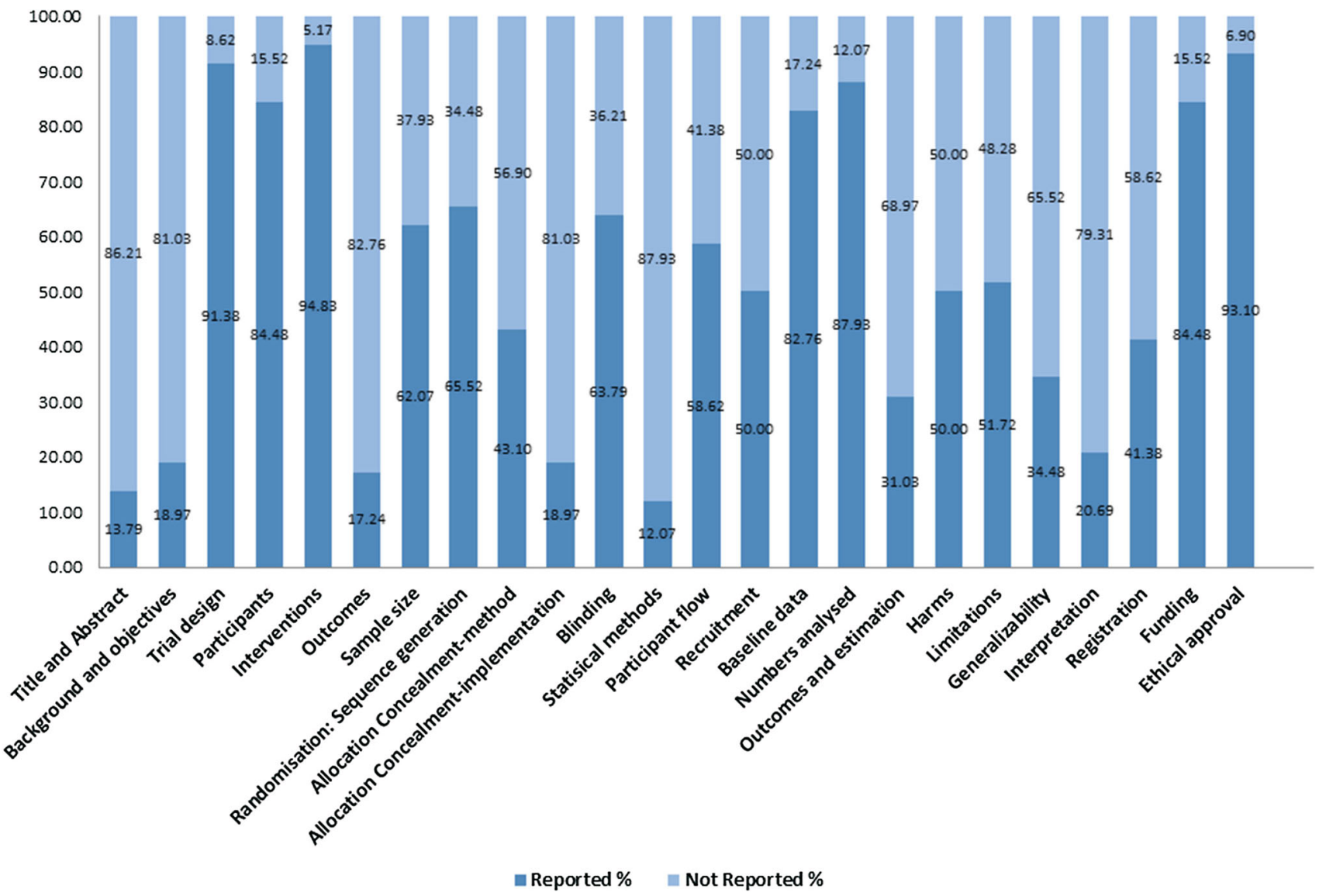

Fig. 3 Consolidated Standards of Reporting Trials pilot extension full-text items reported in percentages 
Table 2 Multivariable regression analysis of predictive factors and CONSORT-pilot extension reporting adherence for abstracts and full texts

\begin{tabular}{|c|c|c|c|c|c|c|}
\hline \multirow[t]{2}{*}{ Factor and its effect } & \multicolumn{2}{|c|}{$\begin{array}{l}\text { Adjusted incidence } \\
\text { rate ratio } \\
99 \% \text { confidence } \\
\text { interval }\end{array}$} & Significance & \multicolumn{2}{|c|}{$\begin{array}{l}\text { Adjusted incidence } \\
\text { rate ratio } \\
99 \% \text { confidence } \\
\text { interval }\end{array}$} & Significance \\
\hline & \multicolumn{3}{|c|}{ ABSTRACT } & \multicolumn{3}{|c|}{ FULL TEXT } \\
\hline \multicolumn{7}{|l|}{ Industry funding } \\
\hline No & 1.21 & 0.94 to 1.54 & 0.0 & 1.16 & 0.94 to 1.44 & 0.07 \\
\hline Yes & 1 & & & 1 & & \\
\hline \multicolumn{7}{|c|}{ Registered as pilot/feasibility study* } \\
\hline No & 0.68 & 0.54 to 0.87 & $<0.001^{*}$ & 0.67 & 0.54 to 0.82 & $<0.001 *$ \\
\hline Yes & 1 & & & 1 & & \\
\hline \multicolumn{7}{|c|}{ Identified as pilot/feasibility in title or abstract* } \\
\hline No & 0.69 & 0.54 to 0.89 & $<0.001^{*}$ & 0.92 & 0.72 to 1.17 & 0.37 \\
\hline Yes & 1 & & & 1 & & \\
\hline \multicolumn{7}{|c|}{ Primary objective/outcome as feasibility and not hypothesis testing } \\
\hline No & 0.98 & 0.78 to 1.22 & 0.82 & 0.77 & 0.63 to 0.93 & $0.001^{*}$ \\
\hline Yes & 1 & & & 1 & & \\
\hline \multicolumn{7}{|l|}{ Journal } \\
\hline BJA & 1.78 & 1.04 to 3.07 & 0.06 & 0.87 & 0.61 to 1.25 & 0.33 \\
\hline Pain & 1.40 & 0.79 to 2.48 & 0.13 & 1.08 & 0.75 to 1.56 & 0.57 \\
\hline Anesthesiology & 1.46 & 0.82 to 2.60 & 0.09 & 1.17 & 0.83 to 1.64 & 0.24 \\
\hline Anesth Analg & 1.59 & 0.94 to 2.70 & 0.02 & 0.94 & 0.68 to 1.29 & 0.59 \\
\hline Anaesthesia & 1 & & & 1 & & \\
\hline
\end{tabular}

Anesth Analg = Anesthesia and Analgesia; BJA = British Journal of Anesthesia. *Significance shown at $P<0.01$ level for abstracts and full texts

estimation for a clinical hypothesis has clear elements, the same may not apply for a pilot trial. We based our judgement as "appropriately reported" if the authors provided any clarification about their sample size based on feasibility objectives and not on hypothesis testing. ${ }^{2}$ Finally, although it is possible that reporting quality could be greater when separately published study protocols are assessed along with their resulting manuscripts, ${ }^{18,33}$ we have to note that adherence to the CONSORT statement and its checklist is for each individual manuscript and not to be considered as a whole for the protocol plus resulting manuscript.

In conclusion, the reporting of pilot trials in leading anesthesia journals is poor for both abstracts and full texts. By being consistent in their policy towards adherence to the CONSORT extension statement on pilot trials and possibly enforcing it as a mandatory requirement, journal editorial boards can encourage and improve reporting standards. Investigators and authors should be aware of the differences in the conduct and reporting of pilot studies.

Conflicts of interest None declared.

Editorial responsibility This submission was handled by Dr. Gregory L. Bryson, Deputy Editor-in-Chief, Canadian Journal of Anesthesia.
Author contributions Harsha Shanthanna was the primary investigator involved in the study design, conduct of the literature search, data extraction and analysis, and manuscript writing. Alka Kaushal was co-investigator involved in the study design, conduct of the literature search, data extraction, and manuscript writing. Lawrence Mbuagbaw was co-investigator involved in the study methodology, supervision of data extraction and analysis, interpretation of the trial, and manuscript writing. Rachel Couban was co-investigator and senior librarian involved with the literature search, data management, and manuscript writing. Jason Busse and Lehana Thabane were co-investigators involved in the study methodology, supervision and interpretation of the trial, and manuscript writing.

Funding disclosure Dr. Shanthanna was supported by the Canadian Institute of Health Research (CIHR) Randomized Controlled Trials mentoring program award between March 2015 and February 2016.

\section{Appendix 1 Search strategy}

Search ((()((((“Anesthesiology”[Journal]) OR “Br J Anaesth"[Journal]) OR “Anaesthesia"[Journal]) OR "Pain"[Journal]) OR “Anesth Analg"[Journal])) AND (((pilot or preliminary or feasibility)) AND (study or trial)))) Filters: published in the last ten years. 
Appendix 2 CONSORT item reporting scores in individual studies

\begin{tabular}{|c|c|c|c|c|c|}
\hline Number & Author & Year & Journal & $\begin{array}{l}\text { CONSORT abstract } \\
\text { score }\{0-15\}[\%]\end{array}$ & $\begin{array}{l}\text { CONSORT full text } \\
\text { score }\{0-24\}[\%]\end{array}$ \\
\hline 1. & Cohen & 2007 & Anesthesiology & $5[35.71]$ & 13 [54.17] \\
\hline 2. & Kok & 2014 & Anaesthesia & $5[35.71]$ & 15 [62.50] \\
\hline 3. & Berger & 2009 & BJA & $5[35.71]$ & 5 [20.83] \\
\hline 4. & Jaura & 2014 & BJA & $6[42.86]$ & 10 [41.67] \\
\hline 5. & Stopee & 2013 & BJA & 10 [71.43] & 14 [58.33] \\
\hline 6. & Goon & 2013 & BJA & 4 [28.57] & 7 [29.17] \\
\hline 7. & Singla & 2015 & Pain & 4 [28.57] & 12 [50.00] \\
\hline 8. & Chang & 2010 & Anesthesiology & 2 [14.29] & 8 [33.33] \\
\hline 9. & Ahola Kohut & 2016 & Pain & 4 [28.57] & 19 [79.17] \\
\hline 10. & Bakri & 2008 & Anesth Analg & 4 [28.57] & 11 [45.83] \\
\hline 11. & Candiotti & 2011 & Anesth Analg & 4 [28.57] & 10 [41.67] \\
\hline 12. & Carson & 2010 & Pain & 5 [35.71] & $12[50.00]$ \\
\hline 13. & Sondergaard & 2010 & BJA & 4 [28.57] & 13 [54.17] \\
\hline 14. & Schulz & 2016 & Anesth Analg & $7[50.00]$ & 8 [33.33] \\
\hline 15. & Martinez & 2015 & BJA & $5[35.71]$ & 13 [54.17] \\
\hline 16. & Al tmimi & 2014 & BJA & 9 [64.29] & 18 [75.00] \\
\hline 17. & Buckley & 2009 & BJA & $5[35.71]$ & 8 [33.33] \\
\hline 18. & Weingarten & 2015 & Anesth Analg & 3 [21.43] & 8 [33.33] \\
\hline 19. & Smith & 2007 & Anesthesiology & $5[35.71]$ & 12 [50.00] \\
\hline 20. & Benger & 2016 & BJA & 8 [57.14] & 20 [83.33] \\
\hline 21. & Goldberg & 2011 & Pain & $6[42.86]$ & 13 [54.17] \\
\hline 22. & Beringer & 2015 & BJA & Not applicable & 14 [58.33] \\
\hline 23. & Short & 2014 & Anesth Analg & $12[85.71]$ & 21 [87.50] \\
\hline 24. & Palermo & 2015 & Pain & 9 [64.29] & 23 [95.83] \\
\hline 25. & Hong & 2008 & Anesth Analg & $7[50.00]$ & $12[50.00]$ \\
\hline 26. & Candiotti & 2007 & Anesth Analg & 4 [28.57] & 10 [41.67] \\
\hline 27. & Parke & 2016 & BJA & 10 [71.43] & 19 [79.17] \\
\hline 28. & Warner & 2015 & Anesthesiology & 3 [21.43] & 15 [62.50] \\
\hline 29. & Olson & 2009 & Anesth Analg & $6[42.86]$ & 13 [54.17] \\
\hline 30. & Chung & 2009 & Anesth Analg & $7[50.00]$ & 16 [66.67] \\
\hline 31. & Blum & 2013 & Anesthesiology & $5[35.71]$ & 18 [75.00] \\
\hline 32. & Zhang & 2012 & Anesth Analg & 5 [35.71] & $9[37.50]$ \\
\hline 33. & Feldheiser & 2102 & BJA & $5[35.71]$ & 15 [62.50] \\
\hline 34. & Manjunath & 2008 & Anesth Analg & 5 [35.71] & 8 [33.33] \\
\hline 35. & Kanai & 2009 & Anesth Analg & $5[35.71]$ & 11 [45.83] \\
\hline 36. & Morelli & 2008 & BJA & $6[42.86]$ & 14 [58.33] \\
\hline 37. & Curry & 2015 & BJA & 13 [92.86] & 23 [95.83] \\
\hline 38. & Dingley & 2015 & Anesth Analg & 3 [21.43] & 8 [33.33] \\
\hline 29. & Morone & 2007 & Pain & $6[42.86]$ & $15[62.50]$ \\
\hline 40. & Thorn & 2011 & Pain & 6 [42.86] & 17 [70.83] \\
\hline 41. & Grunewald & 2014 & BJA & $7[50.00]$ & 14 [58.33] \\
\hline 42. & Chen & 2010 & Anesthesiology & 4 [28.57] & 10 [41.67] \\
\hline 43. & McCohan & 2011 & Anaesthesia & 4 [28.57] & 13 [54.17] \\
\hline 44. & Grossherr & 2009 & BJA & 4 [28.57] & 8 [33.33] \\
\hline 45. & Duan & 2012 & Anesth Analg & $5[35.71]$ & 8 [33.33] \\
\hline 46. & $\mathrm{Wu}$ & 2016 & Anesthesiology & 8 [57.14] & 16 [66.67] \\
\hline
\end{tabular}




\begin{tabular}{|c|c|c|c|c|c|}
\hline Number & Author & Year & Journal & $\begin{array}{l}\text { CONSORT abstract } \\
\text { score }\{0-15\}[\%]\end{array}$ & $\begin{array}{l}\text { CONSORT full text } \\
\text { score }\{0-24\}[\%]\end{array}$ \\
\hline 47. & Sen & 2013 & BJA & Not applicable & $3[12.50]$ \\
\hline 48. & Skelton & 2016 & Anesth Analg & 4 [28.57] & $12[50.00]$ \\
\hline 49. & McElwain & 2008 & Anesth Analg & $7[50.00]$ & 9 [37.50] \\
\hline 50. & Paauwe & 2008 & Anaesthesia & 4 [28.57] & 9 [37.50] \\
\hline 51. & Karkouti & 2012 & Anesthesiology & 6 [42.86] & 19 [79.17] \\
\hline 52. & Papagallo & 2013 & Pain & 4 [28.57] & 15 [62.50] \\
\hline 53. & Cattano & 2012 & BJA & Not applicable & 4 [16.67] \\
\hline 54. & Kothman & 2009 & BJA & $6[42.86]$ & $12[50.00]$ \\
\hline 55. & Rini & 2015 & Pain & $6[42.86]$ & 22 [91.67] \\
\hline 56. & Meierhenrich & 2010 & Anaesthesia & 1 [7.14] & 10 [41.67] \\
\hline 57. & Short & 2011 & Pain & $7[50.00]$ & 14 [58.33] \\
\hline 58. & Deschamps & 2016 & Anesthesiology & 10 [71.43] & 23 [95.83] \\
\hline
\end{tabular}

An59.esth Analg = Anesthesia and Analgesia; BJA= British Journal of Anesthesia; CONSORT = Conso60.lidated Standards of Reporting Trials

\section{Appendix 3 CONSORT Pilot extension items adapted for decision making}

Abstract items

\begin{tabular}{|c|c|c|c|}
\hline Item & Standard checklist item & Extension for pilot trials & $\begin{array}{l}\text { Decision-making rules for items. } \\
\text { CHECK Whether the ITEM WAS } \\
\text { REPORTED OR NOT }\end{array}$ \\
\hline
\end{tabular}

1. Title

2. Trial design

Identification of study as randomized

Description of the trial design (e.g., parallel, cluster, non-inferiority)

\section{METHODS}

3. Participants

4. Interventions

5. Objective

6. Outcome

7. Randomization How participants were allocated to

8. Blinding Whether or not participants, care (masking) interventions

Eligibility criteria for participants and the settings where the data were collected

Interventions intended for each group Specific objective or hypothesis

Clearly defined primary outcome for this report

givers, and those assessing the outcomes were blinded to group assignment
Identification of study as randomized pilot trial

Description of pilot trial design (e.g., parallel, cluster)

Eligibility criteria for participants and the settings where the pilot trial was conducted

Same

Must Have the terms

Pilot/feasibility and randomized-controlled trial

Specific objectives of the pilot They must have a FEASIBILITY OBJECTIVE as trial

Pre-specified assessment or measurement to address the pilot trial objective $(s)^{1}$

Same

Same

If they report hypothesis testing as their main objective, it is noted separately

Must be described at least for (one) the FEASIBILITY OBJECTIVE

If they report hypothesis testing as their main objective, it is noted separately

Reported as randomized

Must: describe who were blinded, and who were not blinded.

Single blinded or double blinded or triple blinded is acceptable

Normally assumed as (if they do not report any specific blinding): single (patient), double (patient and physician) 


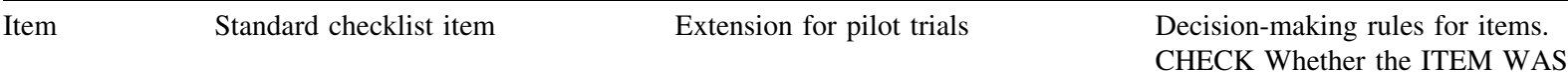

REPORTED OR NOT

RESULTS

9. Numbers randomized

Number of participants randomized to each group

10. Recruitment Trial status (for conference abstracts)

11. Numbers analyzed

12. Outcome

Number of participants analyzed in each group

For the primary outcome, a result for each group and the estimated effect size and its precision

13. Harms

Important adverse events or sideeffects

14. Conclusions General interpretation of the results

15. Trial registration

Registration number and name of trial register

16. Funding

Source of funding
Number of participants screened and randomized to each group for the pilot trial objective $(s)^{1}$

Same

Number of participants analyzed in each group for the pilot objective(s) ${ }^{1}$

Results for the pilot objective(s); including any expressions of uncertainty ${ }^{1}$

Same General interpretation of the results of
pilot trial and their implications for the future definitive trial

Registration number for pilot trial and name of trial register

Source of funding for pilot trial
Clear numbers for participants screened and randomized to each group ( 2 items)

\section{NOT APPLICABLE}

Numbers analyzed for each group compared with numbers randomized

Must: Clear reporting of outcomes with their effect size and uncertainty (both important)

If they report hypothesis testing as their main outcome, it is noted separately

They must report the adverse events that were considered in Methods, or at least mention no adverse effects such as -

2 requirements must be met. On the feasibility success/failure and future trial implications

If it is not registered, they do not fulfil it. They must mention both the number and the trial registry name

They must declare it as: funded or unfunded, and if funded-details of funding

Industry funding is noted separately for either complete trial or part of the trial

\section{Full text items}

\begin{tabular}{|c|c|c|c|}
\hline $\begin{array}{l}\text { Section/topic } \\
\text { and item no. }\end{array}$ & Standard checklist item & Extension for pilot trials & $\begin{array}{l}\text { Decision-making rules for items. } \\
\text { CHECK Whether the ITEM WAS } \\
\text { REPORTED OR NOT }\end{array}$ \\
\hline
\end{tabular}

Title and

abstract

$1 \mathrm{a}$

$1 \mathrm{~b}$
Identification as a randomized trial Identification as a pilot or feasibility in the title

Structured summary of trial design, methods, results, and conclusions (for specific guidance see CONSORT for abstracts) randomized trial in the title

Structured summary of pilot trial design, methods, results, and conclusions (for specific guidance see CONSORT abstract extension for pilot trials)
Must have both fulfilled.

1. Title: Pilot/feasibility and randomizedcontrolled trial

2. Abstract: Design, Methods, Results, and Conclusions.

To facilitate a decision: we require at least one feasibility objective and outcome, and Conclusion must be based on pilot (feasibility) trial not subsequent (clinical) trial 


\begin{tabular}{|c|c|c|c|}
\hline $\begin{array}{l}\text { Section/topic } \\
\text { and item no. }\end{array}$ & Standard checklist item & Extension for pilot trials & $\begin{array}{l}\text { Decision-making rules for items. } \\
\text { CHECK Whether the ITEM WAS } \\
\text { REPORTED OR NOT }\end{array}$ \\
\hline
\end{tabular}

$\begin{array}{lll}\begin{array}{l}\text { Introduction } \\ \text { Background } \\ \text { and } \\ \text { objectives: }\end{array} & \\ 2 \mathrm{a} & \begin{array}{l}\text { Scientific background and explanation of } \\ \text { rationale }\end{array} & \begin{array}{c}\text { Scientific background and explanation of } \\ \text { rationale for future definitive trial, and } \\ \text { reasons for randomized pilot trial }\end{array} \\ 2 \mathrm{~b} & \text { Specific objectives or hypotheses } & \begin{array}{l}\text { Specific objectives or research questions for } \\ \text { pilot trial }\end{array}\end{array}$

Methods

Trial design:

3a

Description of trial design (such as parallel, factorial) including allocation ratio

$3 b$

Important changes to methods after trial commencement (such as eligibility criteria), with reasons

Participants:
$4 \mathrm{a}$
Eligibility criteria for participants
$4 b$
Settings and locations where the data were collected

$4 \mathrm{c}$

Interventions:

5

The interventions for each group with sufficient details to allow replication, including how and when they were actually administered

Outcomes:

$6 a$

Completely defined pre-specified primary and secondary outcome measures, including how and when they were assessed

$6 b$

Any changes to trial outcomes after the trial commenced, with reasons

$6 c$
Description of pilot trial design (such as parallel, factorial) including allocation ratio

Important changes to methods after pilot trial commencement (such as eligibility criteria), with reasons

Must have all the following:

1. Background

2. Rationale for pilot/feasibility

3. Rationale for RCT

4. Objective as at least one feasibility objective clearly outlined

If they report hypothesis testing as their main objective, it is noted separately

Must have the description of design

Design description-parallel, factorial, cluster, crossover; for parallel group it is implied if the description informs of 2 groups

Important changes-if any

Must have all three

1. Eligibility criteria

2. Specific settings such as preop clinic, OR, chronic pain clinic

How participants were identified and consented

3. How was the screening and consent done

Must have

Description of intervention (how and when) enough to allow for replicability

Must have both the following items fulfilled

assessments or measurements to address each pilot trial objective specified in $2 b$, including how and when they were assessed

1. At least one feasibility objective

2. How and when they were measured

If they report hypothesis testing as their main outcome, it is noted separately

Any changes to pilot trial assessments or measurements after the pilot trial commenced, with reasons

If applicable, pre-specified criteria used to judge whether, or how, to proceed with future definitive trial
Any changes if any (not necessary otherwise)

Only if applicable 


\begin{tabular}{|c|c|c|c|}
\hline $\begin{array}{l}\text { Section/topic } \\
\text { and item no. }\end{array}$ & Standard checklist item & Extension for pilot trials & $\begin{array}{l}\text { Decision-making rules for items. } \\
\text { CHECK Whether the ITEM WAS } \\
\text { REPORTED OR NOT }\end{array}$ \\
\hline
\end{tabular}

Sample size:

$7 \mathrm{a}$

How sample size was determined

$7 \mathrm{~b}$

When applicable, explanation of any interim analyses and stopping guidelines

Randomization

Sequence generation:

\section{$8 \mathrm{a}$}

$8 \mathrm{~b}$

Method used to generate the random allocation sequence

Type of randomization; details of any restriction (such as blocking and block size)

Allocation concealment mechanism:

9

Mechanism used to implement the random allocation sequence (such as sequentially numbered containers), describing any steps taken to conceal the sequence until interventions were assigned

Implementation:

10

Who generated the random allocation sequence, who enrolled participants, and who assigned participants to interventions

Blinding:

11a

$11 \mathrm{~b}$
If done, who was blinded after assignment to interventions (for example, participants, care providers, those assessing outcomes) and how

If relevant, description of the similarity of interventions
Rationale for numbers in the pilot trial

Type of randomization(s); details of any restriction (such as blocking and block size)

\section{Must describe}

An explanation of the sample estimate and its basis, even if no statistical calculation

If sample size estimate was based on hypothesis testing of clinical parameters, it is noted separately

Must describe the method used to randomize; just saying randomized is not sufficient

Whether it was appropriately performed or not, this judgement is considered separately as a measure of study methods and noted separately

Allocation concealment: must report how?

Whether it was appropriately performed or not, this judgment, is considered separately as a measure of study methods, and noted separately

Must have all three

1. Who generated the random sequence

2. Who enrolled the patients

3. Who allocated the patients and how

Description of allocation concealment in a way that can be implemented and replicated in the future definitive RCT

Must: describe who were blinded and who were not

Single or double or triple blinded is acceptable.

Normally assumed as (if they do not report any specific blinding): single (patient), double (patient and physician)

Whether it was appropriately performed or not, this judgment, is considered separately as a measure of study methods and noted separately 


\begin{tabular}{|c|c|c|c|}
\hline $\begin{array}{l}\text { Section/topic } \\
\text { and item no. }\end{array}$ & Standard checklist item & Extension for pilot trials & $\begin{array}{l}\text { Decision-making rules for items. } \\
\text { CHECK Whether the ITEM WAS } \\
\text { REPORTED OR NOT }\end{array}$ \\
\hline
\end{tabular}

\section{Analytical \\ methods:}

Results
$\begin{aligned} & \text { Participant flow } \\ & \text { strongly } \\ & \text { recommended): } \\ & \text { 13a }\end{aligned}$
$\begin{aligned} & \text { Methods for additional analyses, such as } \\ & \text { subgroup analyses and adjusted } \\ & \text { analyses }\end{aligned}$
$\begin{aligned} & \text { For each group, the numbers of } \\ & \text { participants who were randomly } \\ & \text { assigned, received intended treatment, } \\ & \text { 14a } \\ & \text { and were analyzed for the primary } \\ & \text { outcome }\end{aligned}$
$\begin{aligned} & \text { For each group, losses and exclusions after } \\ & \text { randomisation, together with reasons }\end{aligned}$
$\begin{aligned} & \text { Dates defining the periods of recruitment } \\ & \text { and follow up }\end{aligned}$
Why the trial ended or was stopped

Baseline data:

\section{5}

Numbers analyzed: 16

Outcomes and estimation:
Statistical methods used to compare group Methods used to address each pilot trial objective whether qualitative or quantitative

For each group, the numbers of participants who were approached and/ or assessed for eligibility, randomly assigned, received intended treatment, and were assessed for each objective

Why the pilot trial ended or was stopped

Report the start of recruitment and end of recruitment

Whether the trial was stopped as planned and if not why?

Must report a table with baseline features

Baseline data

For each objective, number of participants Must describe the following (denominator) included in each analysis. If relevant, these analyses should be by randomized group

The number of participants analyzed for each objective/ outcome reported, randomized by group

Must have the following

For each primary and secondary outcome,
results for each group, and the estimated effect size and its precision (such as 95\% confidence interval)

each objective, results including expressions of uncertainty (such as $95 \%$ confidence interval) for any estimates. If relevant, these results should be by randomized group
Reported outcomes should include expressions of uncertainty

Note: for this item, it is not specific to feasibility objective

This may not be reported, as effect sizes are not informative for pilot trials absolute and relative effect sizes is recommended 


\begin{tabular}{|c|c|c|c|}
\hline $\begin{array}{l}\text { Section/topic } \\
\text { and item no. }\end{array}$ & Standard checklist item & Extension for pilot trials & $\begin{array}{l}\text { Decision-making rules for items. } \\
\text { CHECK Whether the ITEM WAS } \\
\text { REPORTED OR NOT }\end{array}$ \\
\hline
\end{tabular}

\section{Ancillary \\ analyses:}

18

Harms:

19

$19 \mathrm{a}$

Discussion

Limitations:

20

Trial limitations, addressing sources of potential bias, imprecision, and, if relevant, multiplicity of analyses

Generalizability:

21

Generalizability (external validity, applicability) of the trial findings

Interpretation:

22

Interpretation consistent with results, balancing benefits and harms, and considering other relevant evidence

Other information

Registration:

Registration number and name of trial registry

Protocol:

Funding:

25

Sources of funding and other support (such as supply of drugs), role of funders

Where the full trial protocol can be accessed, if available
If relevant, other important unintended consequences

Pilot trial limitations, addressing sources of potential bias and remaining uncertainty about feasibility

Generalizability (applicability) of pilot trial methods and findings to future definitive trial and other studies
Must describe the following

Relevant harms/obvious harms must be reported as either did not occur or if occurred compared for each group
Must describe the limitations in the form of both or either:

Sources of bias; uncertainty about feasibility

Must describe whether the methods and results are applicable for the full trial or other studies

If they describe or generalize based on hypothesis testing result/clinical result, then it is scored as unsatisfactory

Interpretation consistent with pilot trial objectives and findings, balancing potential benefits and harms, and considering other relevant evidence

Implications for progression from pilot to future definitive trial including any proposed amendments

Registration number for pilot trial and name of trial registry

Where the pilot trial protocol can be accessed, if available

Must report the following

Funding as not funded, industry funded, or any other sources of funding

If industry funded, noted separately

Must report

thical approval/research review committee approval confirmed with reference number both the following

mostly on feasibility objectives and not hypothesis testing

2. Implications for progression from pilot to future definitive trial
Registration number and name of registry

Need to check to see if it was registered as pilot/feasibility and noted separately

\section{NOT CONSIDERED}

Ethics approval

CONSORT $=$ Consolidated Standards of Reporting Trials; $\mathrm{OR}=$ operating room $; \mathrm{RCT}=$ randomized-controlled trial 


\section{References}

1. Lancaster GA, Dodd S, Williamson PR. Design and analysis of pilot studies: recommendations for good practice. J Eval Clin Pract 2004; 10: 307-12.

2. Thabane L, Ma J, Chu R, et al. A tutorial on pilot studies: the what, why and how. BMC Med Res Methodol 2010; 10: 1.

3. National Institute for Health Research. [Available from URL: https://www.nihr.ac.uk/glossary?letter=P\&postcategory=-1 (accessed May 2018).

4. Arain M, Campbell MJ, Cooper CL, Lancaster GA. What is a pilot or feasibility study? A review of current practice and editorial policy. BMC Med Res Methodol 2010; 10: 67.

5. Schulz KF, Altman DG, Moher D; CONSORT Group. CONSORT. statement: updated guidelines for reporting parallel group randomized trials. Ann Intern Med 2010; 2010(152): 72632.

6. Can OS, Yilmaz AA, Hasdogan M, et al. Has the quality of abstracts for randomised controlled trials improved since the release of Consolidated Standards of Reporting Trial guideline for abstract reporting? A survey of four high-profile anaesthesia journals. Eur J Anaesthesiol 2011; 28: 485-92.

7. Dolgin E. Publication checklist proposed to boost rigor of pilot trials. Nat Med 2013; 19: 795-6.

8. Friedman L. Commentary: Why we should report results from clinical trial pilot studies. Trials 2013; 14: 14.

9. Shanyinde M, Pickering RM, Weatherall M. Questions asked and answered in pilot and feasibility randomized controlled trials. BMC Med Res Methodol 2011; 11: 117.

10. Eldridge SM, Chan CL, Campbell MJ, et al. CONSORT 2010 statement: extension to randomised pilot and feasibility trials. BMJ 2016; 355: i5239.

11. Samaan Z, Mbuagbaw L, Kosa D, et al. A systematic scoping review of adherence to reporting guidelines in health care literature. J Multidiscip Healthc 2013; 6: 169-88.

12. Borg Debono V, Zhang $S$, Ye $C$, et al. A look at the potential association between PICOT framing of a research question and the quality of reporting of analgesia RCTs. BMC Anesthesiol 2013; 13: 44.

13. Mbuagbaw L, Thabane $M$, Vanniyasingam $T$, et al. Improvement in the quality of abstracts in major clinical journals since CONSORT extension for abstracts: a systematic review. Contemp Clin Trials 2014; 38: 245-50.

14. Rios $L P, Y e C$, Thabane L. Association between framing of the research question using the PICOT format and reporting quality of randomized controlled trials. BMC Med Res Methodol 2010; 10: 11 .

15. Web of Science. In-Cites Journal Citation Reports. Available from URL: https://jcr.incites.thomsonreuters.com/ (accessed May 2018).

16. Kurmis $A P$. Understanding the limitations of the journal impact factor. J Bone Joint Surg Am 2003; 85-A: 2449-54.

17. Viera AJ, Garrett JM. Understanding interobserver agreement: the kappa statistic. Fam Med 2005; 37: 360-3.
18. Borg Debono $V$, Zhang $S$, Ye C, et al. The quality of reporting of RCTs used within a postoperative pain management metaanalysis, using the CONSORT statement. BMC Anesthesiol 2012; 12: 13 .

19. Beringer RM, Guard BC, Hawkins $R$, Segar P. Randomized controlled pilot study: does intraoperative clonidine reduce the incidence of post-hospitalization negative behaviour changes in children who are distressed during induction of general anaesthesia? Br J Anaesth 2015; 115: 795-7.

20. Sen P, Izdes $S$, But A. Effects of sevoflurane and propofol anaesthesia on cerebral oxygenation during normocapnia and mild hypercapnia: a pilot study. Br J Anaesth 2013; 110: 318-9.

21. Cattano D, Corso RM, Altamirano AV, et al. Clinical evaluation of the C-MAC D-Blade videolaryngoscope in severely obese patients: a pilot study. Br J Anaesth 2012; 109: 647-8.

22. Sedgwick $P$. Incidence rate ratio. BMJ 2010; 341: c4804.

23. Moher D, Schulz KF, Altman DG; CONSORT Group. The CONSORT statement: revised recommendations for improving the quality of reports of parallel-group randomized trials. Ann Intern Med 2001; 134: 657-62.

24. CONSORT Transparent Report of Trials. Endorsers Journals and Organizations. Available from URL: http://www.consortstatement.org/about-consort/endorsers (accessed May 2018).

25. Munter NH, Stevanovic A, Rossaint R, Stoppe C, Sanders RD, Coburn $M$. CONSORT item adherence in top ranked anaesthesiology journals in 2011: a retrospective analysis. Eur J Anaesthesiol 2015; 32: 117-25.

26. Mills EJ, Wu P, Gagnier J, Devereaux PJ. The quality of randomized trial reporting in leading medical journals since the revised CONSORT statement. Contemp Clin Trials 2005; 26: 480-7.

27. Peters JP, Hooft L, Grolman W, Stegeman I. Assessment of the quality of reporting of randomised controlled trials in otorhinolaryngologic literature - adherence to the CONSORT statement. PloS One 2015; 10: e0122328.

28. Duffett M, Choong K, Hartling L, Menon K, Thabane L, Cook DJ. Pilot randomized trials in pediatric critical care: a systematic review. Pediatr Crit Care Med 2015; 16: e239-44.

29. De Angelis C, Drazen JM, Frizelle FA, et al. Clinical trial registration: a statement from the International Committee of Medical Journal Editors. N Engl J Med 2004; 351: 1250-1.

30. Reveiz L, Cortes-Jofre M, Asenjo Lobos C, et al. Influence of trial registration on reporting quality of randomized trials: study from highest ranked journals. J Clin Epidemiol 2010; 63: 1216-22.

31. Farquhar CM, Showell MG, Showell EA, et al. Clinical trial registration was not an indicator for low risk of bias. J Clin Epidemiol 2017; 84: 47-53.

32. Bakri MH, Nagem H, Sessler DI, et al. Transdermal oxygen does not improve sternal wound oxygenation in patients recovering from cardiac surgery. Anesth Analg 2008; 106: 1619-26.

33. Pildal J, Chan AW, Hrobjartsson A, Forfang E, Altman DG, Gotzsche PC. Comparison of descriptions of allocation concealment in trial protocols and the published reports: cohort study. BMJ 2005; 330: 1049. 